\title{
Peertechz
}

\section{Analysis of spectrum}

\section{allocation of secondary users}

\section{based on linear cooperative}

spectrum sensing techniques

Received: 26 October, 2020

Accepted: 12 April, 2021

Published: 14 April, 2021

*Corresponding author: Zachary Bosire Omariba, Department of Computer Science, Egerton University, Egerton 20115, Kenya, E-mail: erisob@gmail.com

Keywords: Cooperative spectrum sensing; Primary users; Secondary users; Cognitive radio networks

https://www.peertechzpublications.com

Check for updates in cognitive radio networks

\section{Zachary Bosire Omariba*}

Department of Computer Science, Egerton University, Kenya

\begin{abstract}
This study is based on using the optimization problem of linear cooperative spectrum sensing (CSS) techniques for the analysis and correlation of spectrum allocation to secondary users (SUs) without interfering with the operations of the primary users. To achieve this the system must discover when the licensed users are not using their assigned spectrum so that the spectrum is assigned to the unlicensed users. But when the PUs surfaces the Sus that is holding the spectrum releases it immediately. The probabilities of detection, probability of false alarm, and probability of miss detection msust be kept as low as possible and this is confirmed in our simulation results which indicates that probabilities is kept at $<1$. This indicates that there is improved spectrum efficiency in CSS where the SUs uses the spectrum without interfering with the PUs thus forming the main objective of cooperative spectrum sensing.
\end{abstract}

\section{Introduction}

A cognitive radio (CR) is an intelligent radio technology developed to improve the utilization efficiency of radio spectrum due to the increment in spectrum demand to improve by sensing and sharing the unexploited frequency bands in the radio environment. Due to spectrum scarcity the $\mathrm{CR}$ is introduced to accommodate the ever increase in spectrum demand by automatic detection of available subbands in a wireless spectrum and then changes its transmitter and or receiver parameters to allow more concurrent wireless communications in a given spectrum band at a given location forming a dynamic spectrum management practice [1-3]. Therefore the CR is programmed and configured dynamically so that the transceiver can use the best available sub-bands and allowing the unlicensed users to operate alongside the licensed users by utilizing the unutilized radio frequencies [4]. The Primary users (PUs) are the licensed users whereas the secondary users (SUs) are the unlicensed user who access spectrum opportunistically without interfering with the primary user [5,6]. The cooperative spectrum sensing technique is assumed to be the best technique in the improvement of accuracy of spectrum sensing.

The CR is basically a hybrid technique that involves software defined radio (SDR) for easy configuration of radio system parameter in spread spectrum communications. The $\mathrm{CR}$ functions as an autonomous unit in the communications environment due to which it includes the ability of the transceiver to determine its geographic location, user identification and authorization, encryption and decryption of signals, sensing neighbouring wireless devices in operation, and adjustment of output power and modulation characteristics. Information about the environment is exchanged with the networks the $\mathrm{CR}$ accesses. The CR monitors it performance in real-time as well as reading the radio's outputs in order to determine the radio frequency ( $\mathrm{RF}$ ) environment, channel conditions, link performance, etc. then the $\mathrm{CR}$ adjust the radio settings to deliver the required quality of service (QoS) [6], subject to an appropriate combination of user requirements, operational limitations, and regulatory constraints. According 
to [7], overall network output can be maximized through the use of unlicensed sensor nodes that utilize idle licenced user spectrum which overally improves spectrum use through the exploitation of idle spectrum. With improvements of technology smart radio proposals combine wireless mesh network, CR, and SDR. The mesh network dynamically changes the path messages taken between two given nodes using cooperative diversity; the CR dynamically changes the frequency band used by the messages between two consecutive nodes on the path; and the SDR dynamically changes the protocol used by the message between two consecutive nodes.

There are basically two types of CR i.e. a full CR, and a spectrum-sensing CR (SSCR). A full CR takes into account all parameters that a wireless node or network can be aware of, while SSCR is used to detect free sub-bands or channels in the RF spectrum. The CR technology is very beneficial to the users and the network operators: to the users it reduces the cos of sharing the spectrum which is below the cost of purchasing a licenced band; while to the network operators the CR enhances the user base of a network catering for specific applications since CR technology allows Sus to utilize the unoccupied white spaces of the PU spectrum thus enhancing the system efficiency [8]. The goal of this paper is to analyse the spectrum allocation to SUs, and perform some simulations based on linear cooperative spectrum sensing in cognitive radio networks (CRN). This is done with a view to improve utilization efficiency of radio spectrum due to increased demand of spectrum, and to improve the sensing and sharing of unexploited frequency bands in the radio environment. Section II presents the problem statement, Section III outlines the architecture of CRN. In Section IV the sensing technologies of $\mathrm{CR}$ are discussed. The simulation results and performance analysis is represented in Section $\mathrm{V}$, and the conclusion is provided in Section VI.

\section{Problem statement}

Cognitive radio has aroused a lot of interest as a solution to spectrum scarcity in the next generation of wireless communication. The main idea of cognitive radio is to let the secondary users (SUs) opportunistically access the channels that are temporarily not occupied by the pre-assigned primary users (PUs). In a cognitive radio system, the access priorities of PUs have to be guaranteed, i.e., SUs need to learn the PUs' activities to avoid interfering with the PUs on the band. Therefore, SUs need to sense the PU activity on a particular spectrum before transmitting data on that spectrum. Due to limited sensing capabilities of individual SUs, cooperative spectrum sensing is provided as a way to gather SUs' sensing information in order to increase the accuracy of PU occupancy detection. However, cooperative spectrum sensing can be attacked by malicious SUs, who can intentionally report fake sensing results to mislead the final aggregated result. Our study is aimed to design and develop a technique to analyse and correlate spectrum allocation of secondary users using optimization problem of linear cooperative spectrum sensing techniques.

\section{Architecture of cognitive radio network}

The CR network architecture is composed of a number of primary networks (PNs) that exist with the same coverage are of a single CR network. The PN also known as a licenced network is an existing network which is licenced to operate in a certain spectrum band. The spectrum licenced to the PNs can only be accessed by its users only, as PNs are non-intrusive and the transmissions of the PUs should not be affected by the SUs. The PNs therefore, define the upper bounds on the CR network activities in their licensed bands in terms of maximum power levels to guarantee the promised performance level of the subscribers. The SUs are not licensed to operate in the predefined bands, thus SUs achieve spectrum access in an opportunistic manner and can only utilize the unutilized spectrum [9]. The architecture of the CR network is categorized into two: centralized CR networks, and distributed CR networks. In the centralized CR network the channel sharing is anticipated to permit several CR nodes together to use the unutilized spectrum band for opportunistic transmission. The spectrum in the system is managed by the CR controller (CRS) who fairly assigns the spectrum bands among the users according to their priority order to increase the spectrum capacity in the system. Therefore if the users are assigned priority levels, then the spectrum allocation will take place based on the priority order. If one user enters the queue awaiting to be assigned a spectrum but withdraws before the assignment takes place, then the next user will take that chance in that order. The major challenge faced by the CRC after detecting the available spectrum is sharing the spectrum among the CR users.

As shown in Figure 1, the CR architecture which is commonly known as the cognitive cycle includes detecting spectrum white space, selecting the best frequency bands, coordinating spectrum access with other users and vacating the frequency when a PU appears [10]. Thus spectrum sensing involves sensing of the unused spectrum bands, spectrum sharing involves the management of access channels between many users while averting collision, spectrum decision involves the access and decision of the best channels, and spectrum mobility

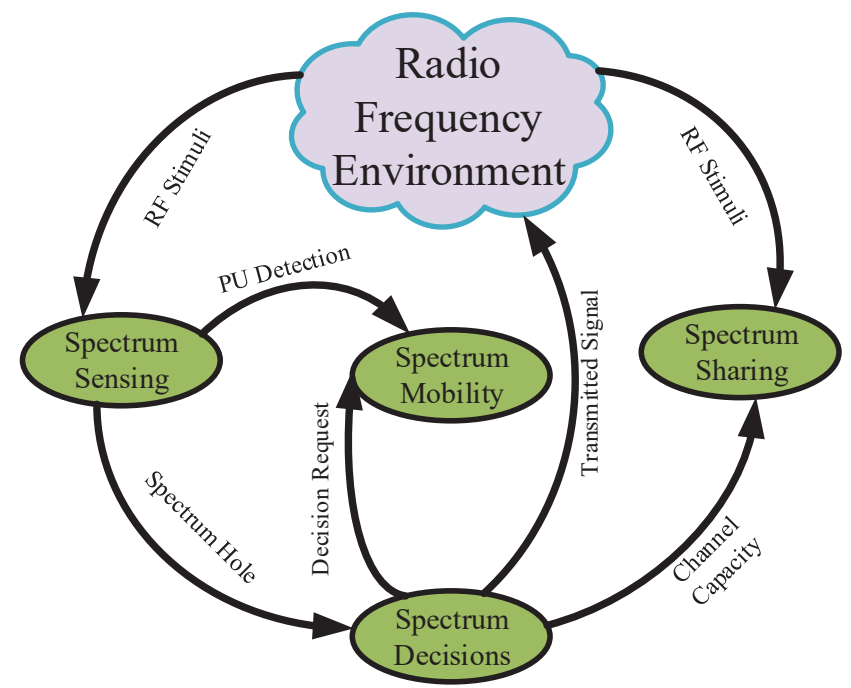

Figure 1: The CR Architecture. 
involves the switching to target channel when a licensed user appears. The cognitive cycle is supported by spectrum sensing and power control functions. The unused spectrum is first detected and shared without harmful interference to other users, and the power control function used for both spectrum access and spectrum sharing CR systems to find the cut-off level of so und-to-noise-ratio (SNR) supporting the channel allocation and imposing interference power constraints for the PUs protection as well $[11,12]$.

The analysis and decision can be supported by learning mechanisms which exploit the knowledge obtained from the execution of prior decisions, while the observation stage typically involves making measurements at several nodes of a CRN. These measurements must be reported to the entity in charge of analysing them to extract the relevant information about the radio environment and to execute the decisionmaking [13]. The SUs needs to sense all vacant frequency bands and the spectrum sensing allows an opportunistic utilization of the unutilized bands. Many researchers have covered broad areas in CR networks spectrum sensing to accomplish the increasing call of more frequency bands and increased traffic handling [9]. The CR technology provides problem solving solution for future wireless networks to utilize available unused frequency bands in an opportunistic appearance, improving the systems performance without interfering with the PUs.

\section{Spectrum sensing}

Spectrum sensing (SS) is fundamentally the most important aspect of $C R$ network management as it calls for timely, speedy, and accurate monitoring of the RF environment to identify the unlicensed bands and then assign them to SUs to enhance $C R$ system efficiency. The spectrum sensing phase is divided into five small phases, sensing phase, reporting phase, CRC phase, re-transmission phase and again $C R C$ for the re-transmitted sensing measurement. In the sensing phase, all cooperative SUs perform local SS simultaneously, while in reporting phase, local sensing data is reported to the FC. In CRC phase, CRC of each SUs is conducted to detect the error. In re-transmission phase, sensing measurements of all SUs on which error greater than threshold is detected are re-transmitted. In data transmission phase, data of SUs is transmitted [14]. Each time the spectrum hole is viewed to ascertain when the PUs is not transmitting, and also to ensure that the one used by the SUs in transmission does not cause interference to all PUs across all frequencies. Thus it is upon the SUs to perform ss either sporadically or successively so that when the PUs reappear they can stop instantly [15][16].

In $C R$, a general model for spectrum sensing can be shown as:

$$
\begin{array}{ll}
x(n)=w(n) & H_{0}: \text { Primary user absence } \\
x(n)=h s(n)+w(n) & H_{1}: \text { Primay user presence }
\end{array}
$$

where $x(n)$ is the signal received by the $C R, w(n)$ is the receiver additive, complex white Gaussian noise (CWGN) which is assumed to be an independent and identically distributed (i.i.d) random process with zero mean and variance $\sigma n^{2}, s(n)$ is samples of transmitted signal generated by the primary transmitter ( $P U$ signal), and $h$ is the complex amplitude gain of the channel; the hypothesis $H_{o}$ and $H_{1}$ represent whether the signal is absent or present for $P U$ detection using $C R$ receiver equipped with single antenna element respectively. Using energy detector, the $C R$ will compare the collected energy $E$ with a predefined threshold $\lambda$ to decide whether the PU channel is occupied or idle.

$$
\begin{aligned}
& E=\Sigma_{1}^{N} x^{2}(n) \\
& \left\{\begin{array}{c}
1, E>\lambda \\
0, \text { otherwise }
\end{array}\right\}
\end{aligned}
$$

The $H_{0}$ hypothesis $H_{1}$ and model is used to implement in various spectrum sensing techniques: matched filter, energy detection, cyclostationary feature detection, and Eigen value based detection. These hypothesis are applied by all spectrum sensing techniques, but they suffer from poor performance under low SNR especially when the AWGN is directly added to the signal. Thus to improve the performance in spectrum sensing techniques, the SNR should be increased, and to achieve this multiple antenna elements or antenna array is utilized at the $C R$ receiver to boost SNR by the value of the array gain [3].

\section{Spectrum management}

When there is inefficient fixed spectrum allocation it leads to scarcity of spectrum and this suggests that there is less usage of licenced spectrum. To find the unoccupied frequency bands is usually difficult due to early allocation of the useful radio spectrum. However in the absence of PU, the SU is allowed to utilize licenced band, without any interference. The CR changes the transmitting parameters and thus adopts easily with the changes in environment, by always identifying the sensing spectrum and identifying the available channels. To achieve this goal the CR network integrates both dynamic spectrum access (DSA) and static spectrum access (SSA) [17]. With the DSA in cognitive radio networks, every senor is made a cognitive sensor node and is enabled to recognize unoccupied channels in overcrowded band as well as to make opportunistic use of the channel for communication. However the priority of the channel use is still belonging to the PUs or licensed user who needs to inform the SUs before it reclaims the channel.

Therefore, the DSA or the unlicensed network usually have CR base station that connects the CR users through single-hop connection for their communication. But, the $\mathrm{CR}$ user requires cognitive functionality to operate in the licensed frequency band with the PUs, since it does not have license and same priority as the PUs to operate in a licensed band. In the case of unlicensed band spectrum operation, a CR user has the same priority to access the unlicensed frequency band as other CR users.

\section{Spectrum sharing}

The sharing of spectrum between the PUs and the SUs in must be realized in CR networks while protecting the PUs 
transmission. This is so because numerous CR users may simultaneously attempt to access the spectrum, thus spectrum sharing ensures that there is smooth and fair competition among CR uses for unlicensed spectrum band so that collision of CR users over corresponding portions of spectrum is averted [13,17]. The spectrum sharing can be vertical or horizontal based on the networks or components involved. When the spectrum sharing takes place within the licensed spectrum bands and licensed user it is termed as vertical spectrum, but if sharing takes place with the unlicensed bands and unlicensed users then it is termed as horizontal spectrum sharing. The spectrum sharing behaviour can be in a cooperative or noncooperative manner.

\section{Cognitive radio sensing techniques}

There are basically two types of CR techniques: noncooperative (transmitter detection) techniques, and cooperative sensing techniques (Figure 2).

\section{Transmitter detection based sensing techniques}

The transmitter detection (non-cooperative) based sensing techniques are the methods that uses the Signal to Noise Ratio (SNR) and the threshold of the detected signal from the transmitter to seek the presence of PUs [18]. However these methods fails to work out robustly whenever the SNR level drops below the required threshold. The transmitter detection based techniques are divided into semi-blind techniques, blind technique, and non-blind technique [19]. The techniques under the transmitter detection based sensing includes energy detection (ED), cyclostationary feature detection (CFD), the matched filter detection(MFD), wavelet spectrum sensing, and the Eigen value based detection. The semi-blind technique does not require any prior information about the signal which needs to be detected (PU), but it needs to know information about noise only so it is known as (semi-blind). An example of a semi-blind technique is the energy detection (ED). The blind technique does not need any information about the PU or noise, so this is called "blind" such as the Wavelet spectrum sensing and Eigen values based detection. The non-blind technique requires all information about both signal detection PU and noise, and many algorithms are built on this technique such as the matched filter detection and cyclostationary feature detection [19].

\section{Cooperative spectrum sensing techniques}

The cooperative spectrum sensing (CSS) is a spectrum sensing technique whose objective is to determine if the spectrums are occupied by licensed users (PUs) or not, so that the unlicensed users (SUs) can utilize that spectrum if it is not occupied [14]. The spectrum hole is sensed and utilized if the Pus is un-occupied, making it very crucial to the performance of both primary and secondary network. The detection performance can be primarily evaluated on the basis of two metrics: probability of false alarm $(\mathrm{PF})$ which reduces the spectral efficiency; and the probability of detection (PD) that avoids the interference with the PUs. The sensing decision of the local secondary user (SU) alone may not be reliable enough due to shadowing, multipath fading and time varying nature of wireless channels between PUs and Sus, and it is due to these challenges that the CSS becomes handy. As many Sus are involved in the CSS, it makes it difficult to achieve the desired performance due to the deficient reporting channel, and the sensing data with error affects the CSS performance.

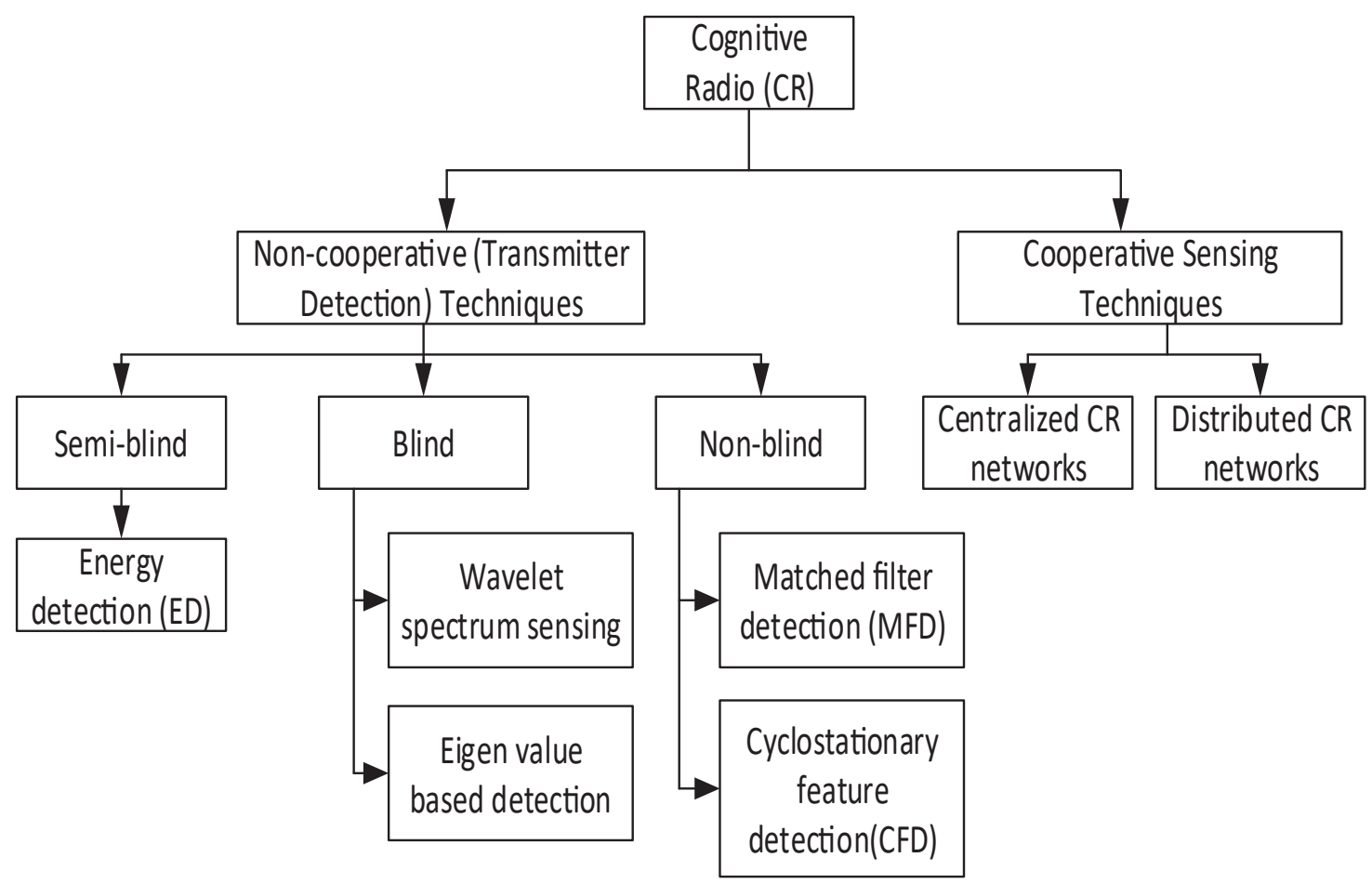


Consequently the existence of large number of Sus involved in the CSS can decrease the energy efficiency of the CR network.

The CSS technique can be divided further into centralized CR network, and distributed CR networks $[20,21]$, to provide the ability to the CR networks to overcome problems related to hidden PUs [22]. In the centralized CR network, SUs report sensing outcomes to a fusion center $(\mathrm{FC})$ and receive instructions from the FC. In a distributed system, SUs do not rely on a DFC for channel access decision making but autonomously decide the channel avail- ability by aggregating outcomes reported by other SUs. Cooperative spectrum sensing is confronted by spectrum sensing data falsification (SSDF) attacks by which malicious SUs to intentionally report fake sensing results to mislead decision making (Figure 3).

In a centralized CSS decision making, a node is selected to act as a FC, for the FC is responsible for deciding the overall $P U$ occupancy. There are many methods used in the centralized CSS which includes soft computing method, hard fusion rule, renewal process method, blind detection method, and weighted average [23]. On the other hand in the distributed CSS decision, the Sus cooperate to detect the spectrum holes in the $C R$ network environment. Unlike in the centralized CSS, here there exists no infrastructure support to act as the fusion

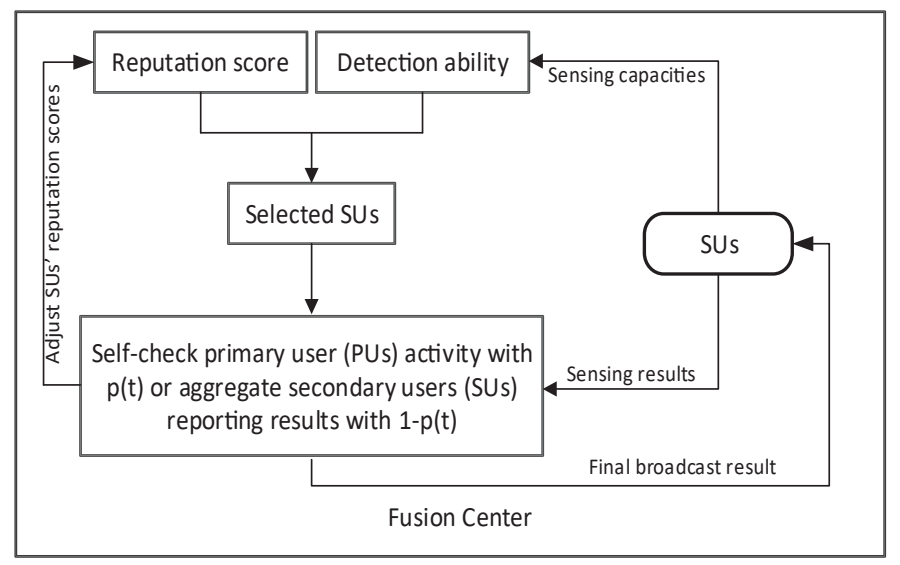

Figure 3: Fusion center architecture of Cooperative sensing spectrum.

Table 1: Strengths and Weaknesses of cooperative spectrum sensing techniques Strengths Weaknesses

provides better performance than single user based spectrum sensing

- improved spectrum utilization

increased levels of agility greater accuracy improved probability of detection

shorter sensing time eliminating of the problem of hidden PUs has a low data throughput because it has to scan a wide range of spectrum cost of overhead performance is often degraded because of fading and shadowing effects

- $\quad$ sensing can be distorted by malicious users who can intentionally report fake sensing results security depletion of ample energy center, as the $S U$ collaborate with its neighbours to determine the presence or absence of the $P U$ signal for the channel of interest (Table 1).

\section{Simulation results and performance analysis}

This section shows the simulations results of CR network and performance analysis based on MATLAB software. The analysis of performance of CSS techniques is basically based on probability of detection $P_{d}$, probability of false alarm $P_{F A}$ and the probability of miss detection $P_{M D}$ (Figure 4).

To discover the availability of a licensed user at times when he is using the spectrum it called $P_{d}$ whereas to discover the false declaration of the presence of licensed users in the spectrum band and $P_{M D}$ is declaring the absence of Pus at the same time of spectrum occupancy. The probability of false alarm $P_{F A}$ will cause utilization of spectrum while the $P_{M D}$ will lead to the interference with PUs, thus the CR network should exhibit low levels of both of these probabilities as possible. The simulation results confirm this fact of keeping the probabilities as low as possible and for these case it is kept at $<1$ are shown in Figures 5 to 15 respectively. Hence it is proved by simulation analysis that CSS scheme provides a better detection probability, less false alarms and lower miss detection of a PU. Therefore a cognitive radio user who is unlicensed can use the spectrum efficiently without interfering PU, which is a main objective of cooperative spectrum sensing in CRN.

From Figure $4(\mathrm{a})-(\mathrm{d})$, where thet $S N R \leq-5 d B, t$ can be shown that the performance is high at low SNR ranges, and performance is low for high SNR rangesas $0 \leq S N R \leq 20 \mathrm{~dB}$ seen in Figure 4(e-i). From Figure 5, there is high probability detection witnessed when the SNR range is high under both averaging filter and the rational IR filter. The averaging filter is least when there is least SNR value ranges between $(-20 \mathrm{~dB}$ to $-10 \mathrm{~dB})$, but this value start to accelerate higher than the rational IR filter as recorded in the simulation experiments (Figures 5-15).

Equally the theoretical probability detection as shown in Figure 5, shows that the value of $P_{d}$ grows exponentially as the SNR values increase between ( $-20 \mathrm{~dB}$ to $5 \mathrm{~dB})$, but this value of $P_{d}$ remains constant for the corresponding SNR values (10dB to $20 \mathrm{~dB}$ ) respectively. The simulation parameters are shown in Table 2.

Thus from Figure 5 and Figure 6, it can be concluded that to improve the $P_{D}$, cooperative SUs needs to be increased. The performance of the energy detection is measured by $P_{d}$ which defines the probability of detecting the presence of the $P U$ signal by the sensing technique given some threshold $\lambda$. The $P_{d}$ can be defined also as the probability of the $P U$ transmitting on the licenced channel being true, and the detector detecting the $P U$ signal. From Figure 6, the probability of false alarm $\left(P_{F A}\right)$ is high when the SNR value is low, and vice versa, and the same case observed in Figure 7, where the probability of miss $\left(P_{M}\right)$ under averaging filter is high when the SNR value is low, but 

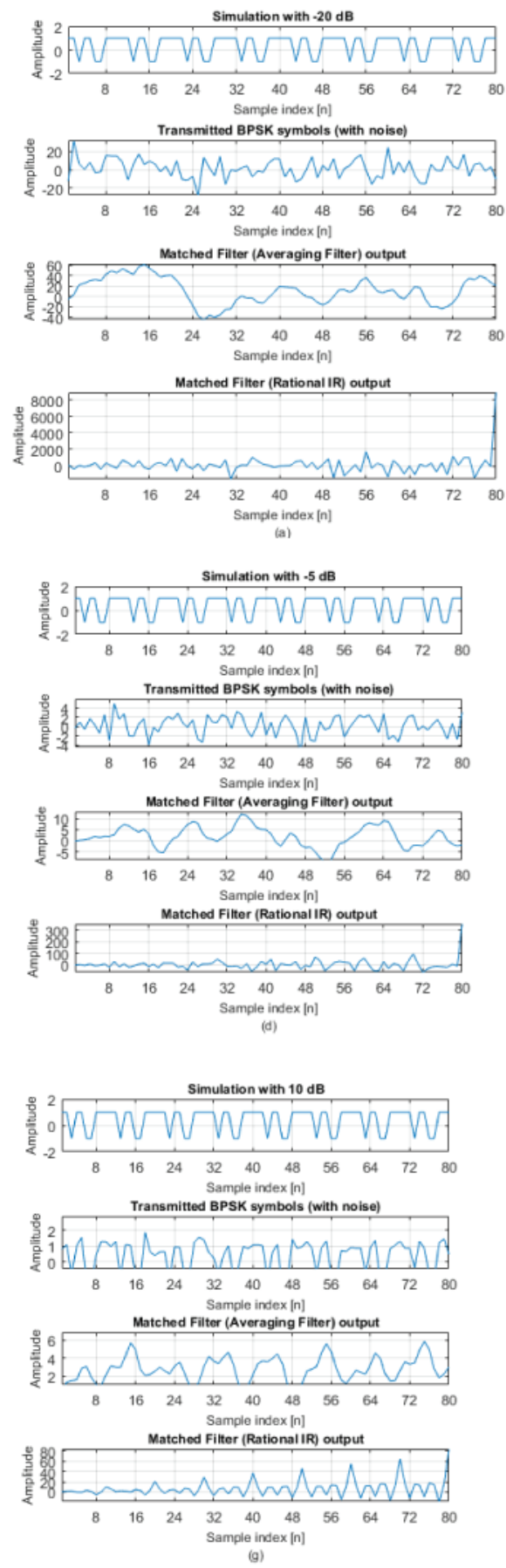
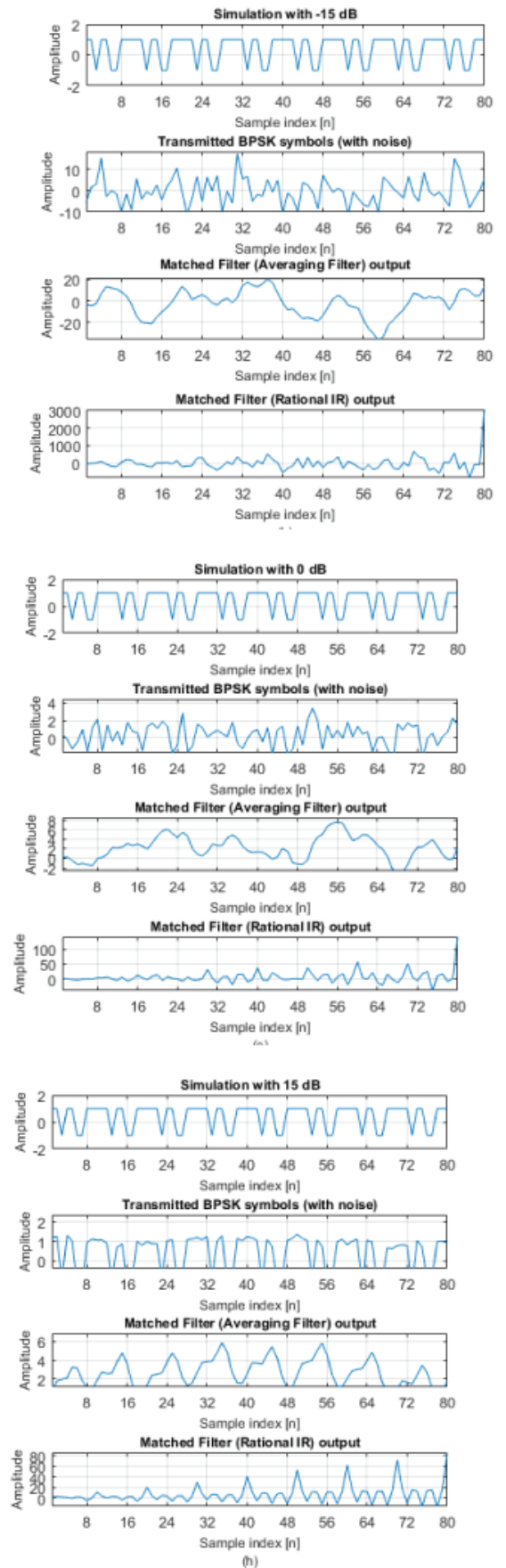
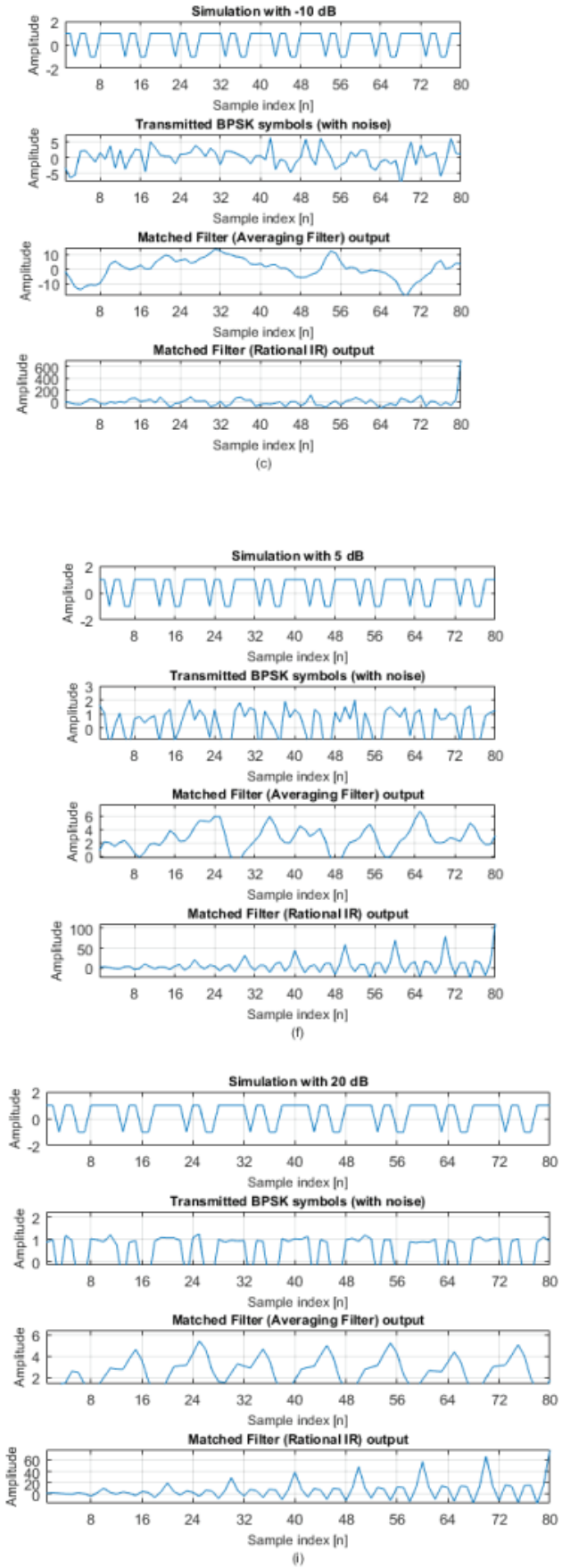

Figure 4: (a)-(i) Comparison of different signal intensities in deciBel (dB)

as the SNR value improves, the $\left(P_{M}\right)$ is high as compared to rational IR filter. In general it is observed that the $\left(P_{M}\right)$ drops as the SNR value increases.

\section{Conclusion}

In literature there are different cooperative detection techniques given for spectrum sensing. The detection probabilities in CRN may also be improved by cooperation among the cognitive radio users (CRUs) but at additional overhead. In this paper, we introduce the linear CSS technique for the analysis of spectrum allocation of SUs and we performed the simulations which is the main objective. The maximum spectrum usage and efficient performance of the CRN is achieved by the application of CSS. This is confirmed in the simulation results that indicates the probability of detection, probability of false alarm, and probability of miss detection kept as low as $<1$. We explore the CSS because of the high efficiency as compared to the non-cooperative spectrum sensing. As shown from the simulation there is improved detection of performance of CSS when the $P_{F A}$ is higher than the $P_{M D}$. Our future work involves an in depth analysis of SNR on each of the detection techniques of spectrum allocation towards improvement of detection performance.

Citation: Omariba ZB (2021) Analysis of spectrum allocation of secondary users based on linear cooperative spectrum sensing techniques in cognitive radio networks. Trends Comput Sci Inf Technol 6(1): 025-033. DOI: https://dx.doi.org/10.17352/tcsit.000035 


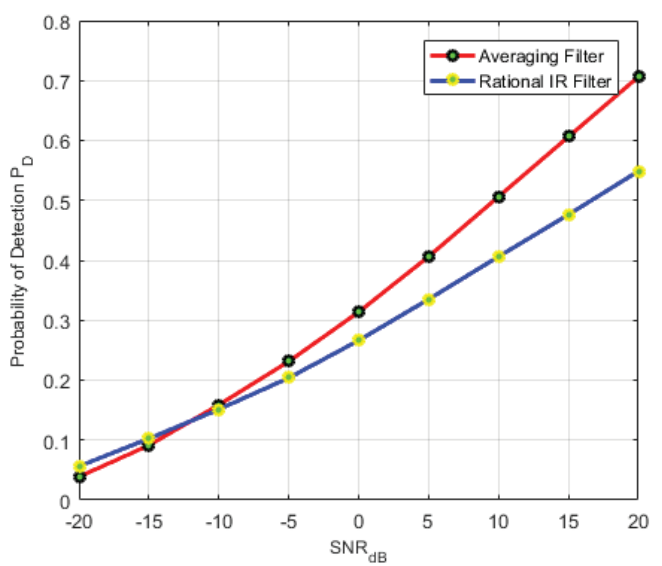

Figure 5: Probability of detection.

Table 2: Simulation Parameters

\begin{tabular}{|c|c|}
\hline Parameter & Value \\
\hline Time index & {$[0,80]$} \\
\hline $\mathrm{P}_{\mathrm{D}}$ & {$[0,1]$} \\
\hline SNR & {$[-20,20] \mathrm{dB}$} \\
\hline Threshold & 1.28 \\
\hline Time slot & $0.001-0.01$ seconds \\
\hline Simulation time & 1000 seconds \\
\hline Fading & Rayleigh \\
\hline
\end{tabular}

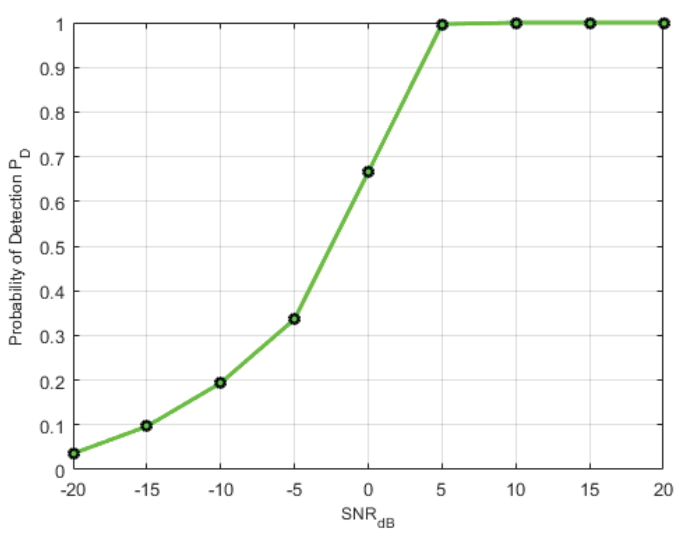

Figure 6: Theoretical Probability of Detection.

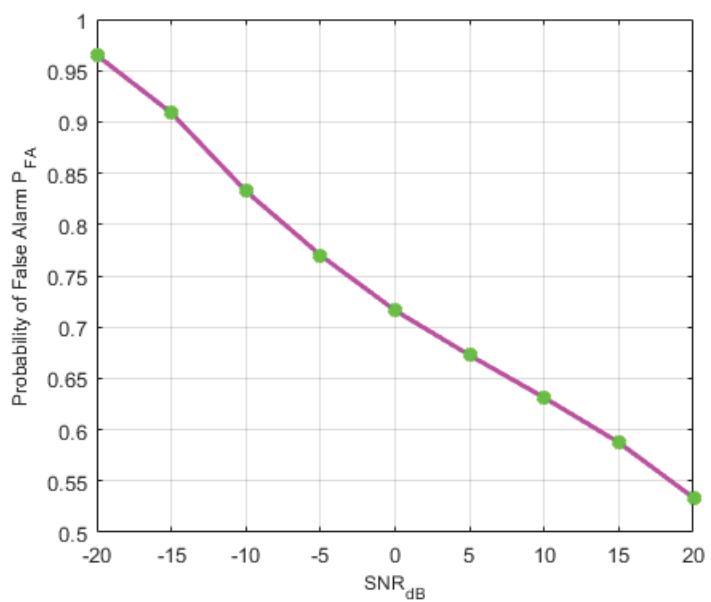

Figure 7: Probability of False Alarm.

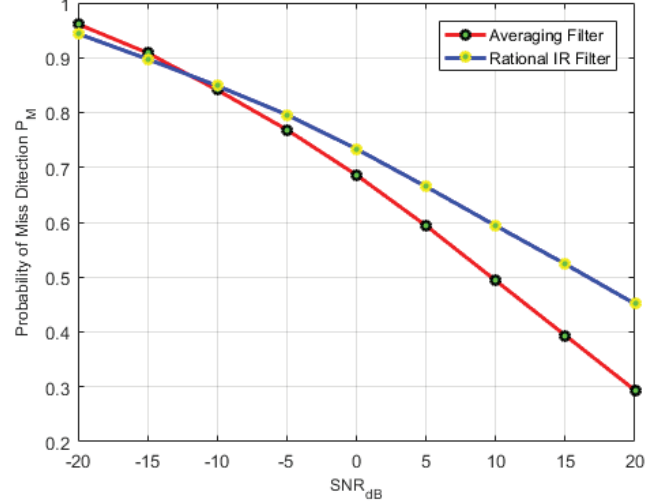

Figure 8: Probability of miss detection.

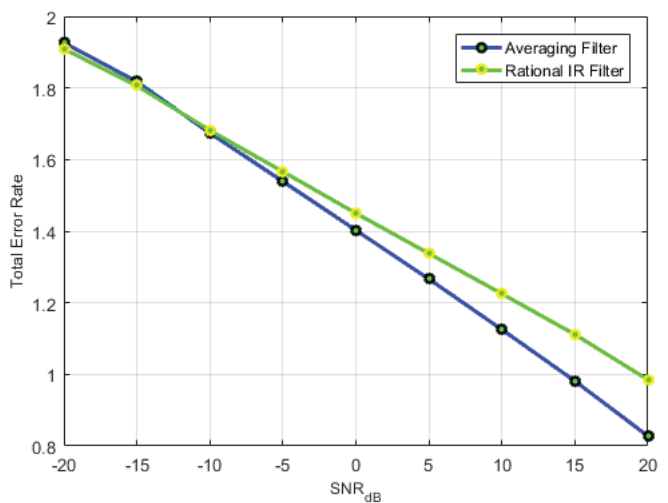

\section{Figure 9: Total error-rate.}

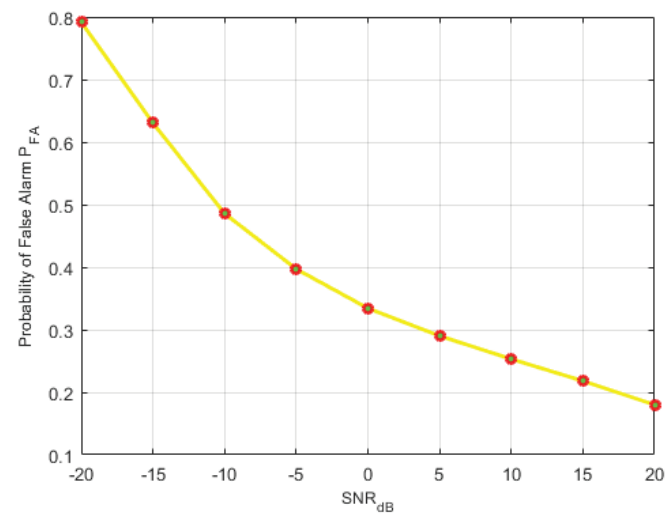

Figure 10: Theoretical Probability of False-alarm

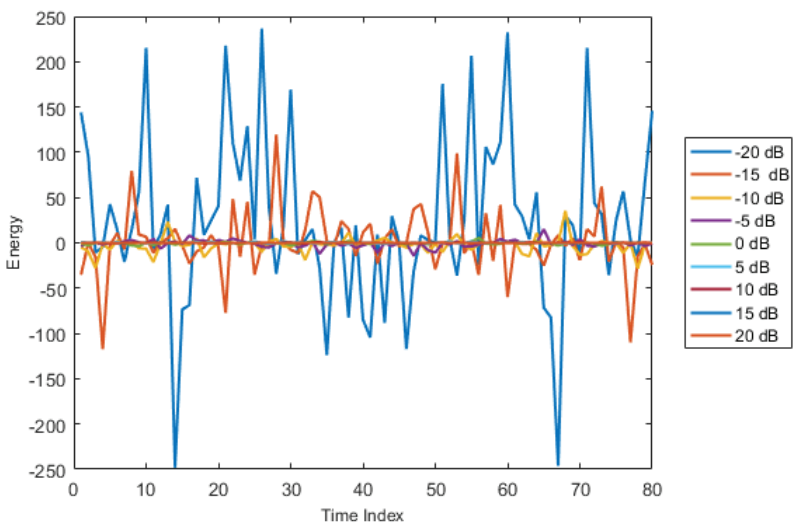

Figure 11: Comparison of simulated dynamic thresholds of different signal strengths.

Citation: Omariba ZB (2021) Analysis of spectrum allocation of secondary users based on linear cooperative spectrum sensing techniques in cognitive radio networks. Trends Comput Sci Inf Technol 6(1): 025-033. DOI: https://dx.doi.org/10.17352/tcsit.000035 


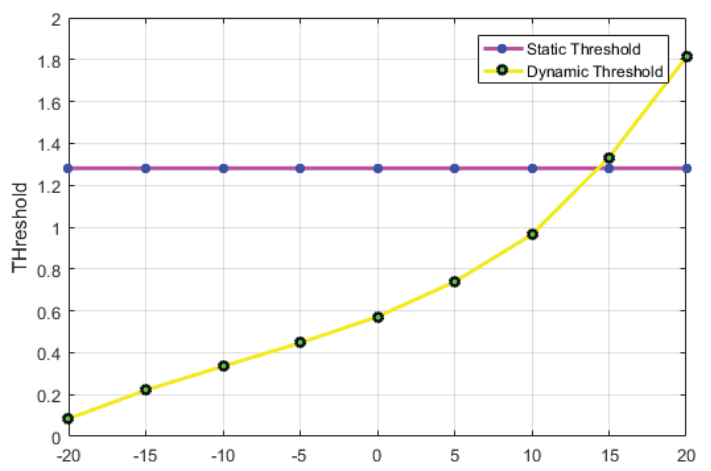

Figure 12: Static and Dynamic Thresholds.

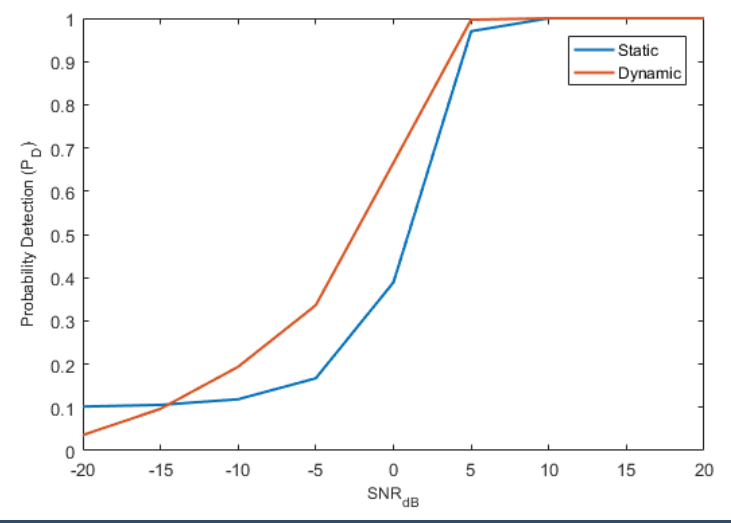

Figure 13: Static vs. Dynamic Probability Detectionv.

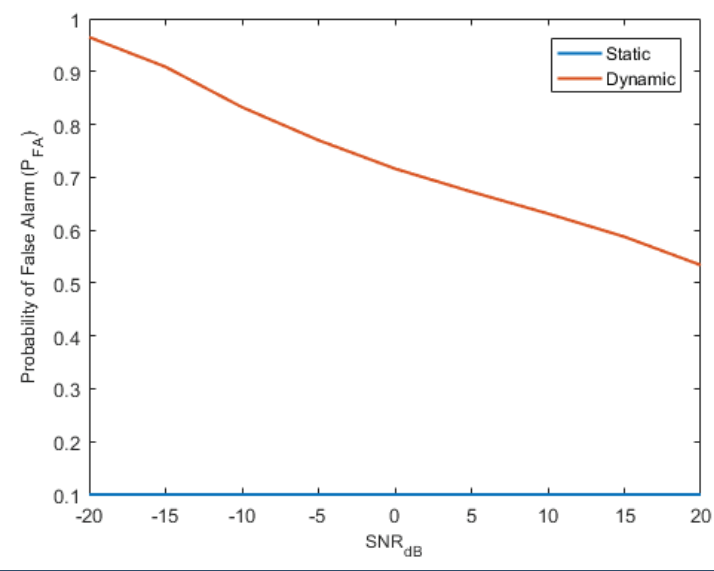

Figure 14: Static vs. Dynamic Probability of False Alarm.

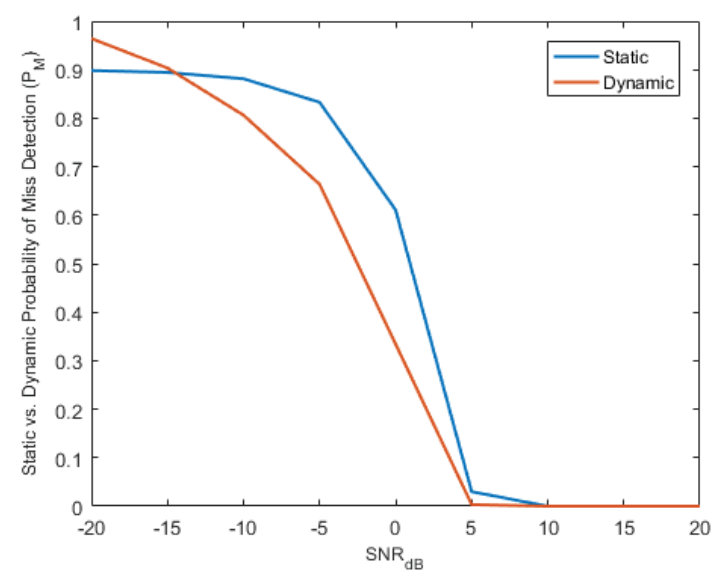

Figure 15: Static vs. Dynamic Probability of Miss Detection.

\section{References}

1. Mourougayane K, Amgothu B, Bhagat S, Srikanth S (2019) A robust multistage spectrum sensing model for cognitive radio applications. Int $\mathrm{J}$ Electron Commun (AEU) 110: 1-11. Link: https://bit.ly/3dYMzfR

2. Khasawneh M, Agarwal A (2016)A Collaborative Approach towards Securing Spectrum Sensing in Cognitive Radio Networks. Procedia Comput Sci 94: 302309. Link: https://bit.ly/3a08dPp

3. Kabeel AA, Hussein AH, Khalaf AAM, Hamed HFA (20190 A utilization of multiple antenna elements for matched filter based spectrum sensing performance enhancement in cognitive radio system. Int J Electron Commun 107: 98-109. Link: https://bit.ly/325QguD

4. Darabkh KA, Amro OM, Bany Salameh H, Al-Zubi RT (2019) A-Z overview of the in-band full-duplex cognitive radio networks. Comput Commun 145: 66-95. Link: https://bit.ly/3s4jWTu

5. Suguna R, Rathinasabapathy $V$ (2019) An SoC architecture for energy detection based spectrum sensing using Low Latency Column Bit Compressed (LLCBC) MAC in cognitive radio wireless sensor networks. Microprocess Microsyst 69: 159-167. Link: https://bit.ly/3taUkFD

6. Ridouani M, Hayar A, Haqiq A (2017) Perform sensing and transmission in parallel in cognitive radio systems: Spectrum and energy efficiency. Digit Signal Process A Rev J 62: 65-80. Link: https://bit.ly/3mCQwL0

7. Bayrakdar ME (2020) Exploiting cognitive wireless nodes for priority-based data communication in terrestrial sensor networks. ETRI J 42: 36-45. Link: https://bit.ly/3uNDT2J

8. Manna T, Misra IS (2019) Design, implementation and analysis of cognitive radio enabled intelligent WBAN gateway for cost-efficient remote health monitoring. Phys Commun 35. Link: https://bit.ly/3teLRRZ

9. Gupta MS, Kumar K (2019) Progression on spectrum sensing for cognitive radio networks: A survey, classification, challenges and future research issues. J Netw Comput Appl 143: 47-76. Link: https://bit.ly/3uNDZY9

10. Nareshkumar S, Bikshalu K (2019) Adaptive absolute SCORE algorithm for spectrum sensing in cognitive radio. Microprocess Microsyst 69: 43-53. Link: https://bit.ly/3uEHLmz

11. Suseela B, Sivakumar D (2015) Non-cooperative spectrum sensing techniques in cognitive radio-a survey, in 2015 IEEE International Conference on Technological Innovations in ICT for Agriculture and Rural Development, TIAR 2015. 2015: 127-133. Link: https://bit.ly/3t9ybYo

12. Amrutha V, Karthikeyan KV (2017) Spectrum sensing methodologies in cognitive radio networks: A survey, in Proceedings of IEEE International Conference on Innovations in Electrical, Electronics, Instrumentation and Media Technology, ICIEEIMT 2017. 2017: 306-310. Link: https://bit.ly/3tc2Xjw

13. Raschellà A, Umbert A (2016) Implementation of Cognitive Radio Networks to evaluate spectrum management strategies in real-time. Comput Commun 79: 37-52. Link: https://bit.ly/3wQVA31

14. Bhatti DMS, Ahmed S, Saeed N, Shaikh B (2018) Efficient error detection in soft data fusion for cooperative spectrum sensing. Int $J$ Electron Commun (AEU) 88: 141-147. Link: https://bit.ly/3t9ynXC

15. Abdul Salam AO, Sheriff RE, Al-Araji SR, Mezher K, Nasir Q (2019) Adaptive threshold and optimal frame duration for multi-taper spectrum sensing in cognitive radio. ICT Express 5: 31-36. Link: https://bit.ly/2RoB9KB

16. Shbat M, Tuzlukov V (2019) Primary signal detection algorithms for spectrum sensing at low SNR over fading channels in cognitive radio. Digit Signal Process 93: 187-207. Link: https://bit.ly/328idSG

17. Oyewobi SS, Hancke GP (2017) A survey of cognitive radio handoff schemes, challenges and issues for industrial wireless sensor networks (CR-IWSN). J Netw Comput Appl 97: 140-156. Link: https://bit.ly/3alqffx

Citation: Omariba ZB (2021) Analysis of spectrum allocation of secondary users based on linear cooperative spectrum sensing techniques in cognitive radio networks. Trends Comput Sci Inf Technol 6(1): 025-033. DOI: https://dx.doi.org/10.17352/tcsit.000035 
18. Ansari AH, Gulhane SM (2016) Investigation of ROC parameters using Monte Carlo simulation in cyclostationary and energy detection spectrum sensing," in Proceedings - IEEE International Conference on Information Processing, ICIP 2015. 2016: 266-271. Link: https://bit.ly/3sbzyo4

19. Fouda MA, Eldien AST, Mansour HAK (2018) FPGA based energy detection spectrum sensing for cognitive radios under noise uncertainty. in Proceedings of ICCES 2017 12th International Conference on Computer Engineering and Systems 2018: 584-591. Link: https://bit.ly/3g3t48E

20. Wang J, Chen IR, Tsai JJP, Wang DC (2018) Trust-based mechanism design for cooperative spectrum sensing in cognitive radio networks. Comput Commun 116: 90-100. Link: https://bit.ly/3wPOpbm

21. Kapoor S, Rao SVRK, Singh G (2011) Opportunistic spectrum sensing by employing matched filter in cognitive radio network," in Proceedings 2011 International Conference on Communication Systems and Network Technologies, CSNT 2011: 580-583. Link: https://bit.ly/3dVjBgL

22. Akhtar R, Rashdi A, Ghafoor A (2009) Grouping technique for cooperative spectrum sensing in cognitive radios," in 2009 2nd International Workshop on Cognitive Radio and Advanced Spectrum Management, CogART 2009: 80-85. Link: https://bit.ly/3mLy96H
23. Chembe C, Noor RM, Ahmedy I, Oche M, Kunda Det al. (2017) Spectrum sensing in cognitive vehicular network: State-of-Art, challenges and open issues. Comput Commun 97: 15-30. Link: https://bit.ly/3wH6icm

24. Dibal PY, Onwuka EN, Agajo J, Alenoghena CO (2018) Algorithm for spectrum hole identification in cognitive radio network based on discrete wavelet packet transform enhanced with Hilbert transform. Comput Commun 125: 1-12. Link: Link: https://bit.ly/3d8aREQ

25. Xie J, Chen J, Wu D (2012) Cooperative spectrum sensing for cognitive radios over fading channels, in Proceedings of 2nd International Conference on Computer Science and Network Technology, ICCSNT 2012: 1962-1966. Link: https://bit.ly/3teMyuz

26. Rasheed T, Rashdi A, Akhtar AN (2018) Cooperative spectrum sensing using fuzzy logic for cognitive radio network," in 2018 Advances in Science and Engineering Technology International Conferences, (ASET), 2018: 1-6. Link:

27. Chaudhary A, Dongre M, Patil H (2016) Energy-Decisive and Upgrade Cooperative Spectrum Sensing in Cognitive Radio Networks. Procedia Comput Sci 79: 683-691. Link: https://bit.ly/3wQWo83

\section{Discover a bigger Impact and Visibility of your article publication with} Peertechz Publications

\author{
Highlights \\ - Signatory publisher of ORCID \\ * Signatory Publisher of DORA (San Francisco Declaration on Research Assessment) \\ * Articles archived in worlds' renowned service providers such as Portico, CNKI, AGRIS, \\ TDNet, Base (Bielefeld University Library), CrossRef, Scilit, J-Gate etc. \\ * Journals indexed in ICMJE, SHERPA/ROMEO, Google Scholar etc. \\ * OAI-PMH (Open Archives Initiative Protocol for Metadata Harvesting) \\ * Dedicated Editorial Board for every journal \\ * Accurate and rapid peer-review process \\ * Increased citations of published articles through promotions \\ - Reduced timeline for article publication \\ Submit your articles and experience a new surge in publication services \\ (https://www.peertechz.com/submission).
}

Peertechz journals wishes everlasting success in your every endeavours.

Copyright: @ 2021 Omariba ZB. This is an open-access article distributed under the terms of the Creative Commons Attribution License, which permits unrestricted use distribution, and reproduction in any medium, provided the original author and source are credited. 